\title{
Acute Ketamine Administration Alters the Brain Responses to Executive Demands in a Verbal Working Memory Task: an fMRI Study
}

\author{
RAE Honey', GD Honey', C O'Loughlin', SR Sharar², D Kumaran', ET Bullmore', DK Menon ${ }^{3}$, T Donovan $^{4}$, \\ VC Lupson ${ }^{5}, \mathrm{R}$ Bisbrown-Chippendale ${ }^{5}$ and PC Fletcher*, \\ 'Department of Psychiatry, University of Cambridge, Cambridge, UK; ${ }^{2}$ Harborview Medical Center, Department of Anesthesiology, University of \\ Washington, Washington, DC, USA; ${ }^{3}$ Department of Anaesthesia, University of Cambridge, Cambridge, UK; ${ }^{4}$ Department of Radiography and \\ Imaging Sciences, St Martin's College, Lancaster, USA; ${ }^{5}$ Wolfson Brain Imaging Centre, University of Cambridge, Cambridge, UK
}

\begin{abstract}
We have used functional MRI to determine the effects of ketamine on brain systems activated in association with a working memory task. Healthy volunteers received intravenous infusions of placebo, ketamine at $50 \mathrm{ng} / \mathrm{ml}$ plasma concentration, and ketamine at $100 \mathrm{ng} / \mathrm{ml}$. They were scanned while carrying out a verbal working memory task in which we varied the executive requirements (manipulation vs maintenance processes) and the mnemonic load (three vs five presented letters). We previously showed that ketamine produces a specific behavioral impairment in the manipulation task. In the current study, we modified tasks in order to match performance across drug and placebo conditions, and used an event-related fMRI design, allowing us to remove unsuccessful trials from the analysis. Our results suggest a task-specific effect of ketamine on working memory in a brain system comprising frontal cortex, parietal cortex, and putamen. When subjects are required to manipulate presented letters into alphabetical order, as opposed to maintaining them in the order in which they were presented, ketamine is associated with significantly greater activity in this system, even under these performance-matched conditions. No significant effect of ketamine was seen in association with increasing load. This suggests that our findings are not explicable in terms of a nonspecific effect of ketamine when task difficulty is increased. Rather, our findings provide evidence that the predominant effects of low, subdissociative doses of ketamine are upon the control processes engaged by the manipulation task. Furthermore, we have shown that ketamine's effects may be elucidated by $\mathrm{M} M \mathrm{R}$ l even when overt behavioral measures show no evidence of impairment.

Neuropsychopharmacology (2004) 29, 1203-1214, advance online publication, 2 I April 2004; doi: I0.1038/sj.npp. I 300438
\end{abstract}

Keywords: ketamine; schizophrenia; working memory; psychopharmacology; fMRI

\section{INTRODUCTION}

The NMDA receptor hypofunction (NRH) model of schizophrenia is supported by remarkably schizophrenialike psychotic and cognitive symptoms following administration of NMDA antagonists, such as PCP and ketamine (Carlsson et al, 1999; Hirsch et al, 1997; Javitt and Zukin, 1991; Jentsch and Roth, 1999; Krystal et al, 1999; Tamminga, 1998). Schizophrenia is a condition characterized by widespread cognitive impairment (Gold and Harvey, 1993) and an important challenge in assessing the ketamine model is

\footnotetext{
*Correspondence: PC Fletcher, Department of Psychiatry, University of Cambridge, Box 255, Addenbrooke's Hospital, Cambridge CB2 2QQ, UK, Tel: + 44 I223 336 988, Fax: + 44 I223336 58I,

E-mail: pcf22@cam.ac.uk

Received OI October 2003; revised 0 I December 2003; accepted 06 February 2004

Online publication: 16 February 2004 at http://www.acnp.org/citations/ Npp081903030048/default.pdf
}

in defining and specifying more fully the effects of the drug in cognitive domains relevant to the disease. The current study has this goal in view. It was based upon previous findings that ketamine is associated with a process-specific deficit in working memory (Honey et al, 2003). Our earlier study showed that, as a working memory task becomes more demanding, low-dose ketamine produces an impairment in subjects' abilities to reorganize, rather than simply to maintain, the contents of working memory. The current study used fMRI to determine whether, at the same doses, but at lower levels of demand (in which there was no apparent performance deficit) a similar dissociation would be observed in the brain's responses, as estimated by the BOLD signal.

A clearer understanding of the influence of ketamine upon working memory is critical in assessing its validity as a model of schizophrenia. Working memory deficits are characteristic of the illness (Adler et al, 1998; Bressi et al, 1996; Conklin et al, 2000; Gooding and Tallent, 2002; 
Mahurin et al, 1998; Morice and Delahunty, 1996; Pantelis et al, 1997; Park and Holzman, 1992; Tek et al, 2002) and functional neuroimaging studies of patients with schizophrenia have implicated frontal regions (Callicott et al, 1998; Carter et al, 1998; Curtis et al, 1998; Manoach et al, 1999, 2000; Menon et al, 2001), which are known to be important in mediating working memory (Braver et al, 1997; Cohen et al, 1997; D’Esposito et al, 1999; De Zubicaray et al, 1998; Friedman and Goldman-Rakic, 1994; GoldmanRakic, 1996; Postle et al, 1999; Smith et al, 1996). The influence of ketamine upon working memory-induced frontal function is clearly, therefore, a matter of interest.

By varying the amount of information to be maintained and/or manipulated, our task allowed us to explore the physiological correlates of manipulation processes and of increasing memory load. Manipulation or executive processes can be distinguished from maintenance in that manipulation involves a task, such as reordering or updating information, which is performed on information being stored in working memory. Maintenance of information simply requires the participant to store information over a short period of time (Baddeley, 1986; Baddeley and Hitch, 1994). We used a factorial design derived from previous fMRI work (D'Esposito et al, 1999). The first factor was working memory load, which was varied across two levels (3 vs 5 items). The second was the type of task subjects were required to carry out: (manipulation of material $v s$ maintenance). We were thus able to explore the effects of an increase in maintenance demands (produced by an increase in load from 3 to 5 items), and of an independently controlled requirement to manipulate information and of the interaction between these two factors. We determined the effects of ketamine (at two doses) on the patterns of brain activation accompanying these cognitive demands. Event-related fMRI was used, allowing us to dispense with incorrect trials in the subsequent analysis and, therefore, to interpret drug-related changes in task-specific activation under balanced performance levels. Our goal was to identify the physiological effects of ketamine that may precede the overt behavioral changes observed when these tasks are made more demanding.

We hypothesized that both increases in memory load and a requirement to manipulate presented material would be associated with an increase in activity within the frontoparietal working memory network, especially. The latter prediction draws upon findings from a previous study exploring the effects of manipulation demands (D'Esposito et al, 1999) and from working memory studies in which comparable demands have been made (see Fletcher and Henson, 2001 for a review). Having established, in our previous study, that, with increasing task demands, ketamine impairs a subject's abilities to manipulate material, we speculated that the effects of ketamine may be observed 'prebehaviorally' in the manipulation task.

\section{METHOD}

\section{Participants}

A total of 12 healthy volunteers (six male, six female; mean age 31.17 years (range 18-49)) participated. The mean IQ of participants was 104.67 (SD 12.71) estimated by the National Adult Reading Test (NART; Nelson, 1991). All participants had a body mass index within $10 \%$ of the ideal weight, and all spoke English as their primary language. Participants were screened for the presence and history of psychotic symptoms, as well as family history of psychosis. They were screened for any drug or alcohol abuse. It transpired that none were smokers. Participants were reimbursed for travel costs and their time. Informed written consent was obtained from all participants. The study was approved by the Local Research Ethics Committee of Addenbrookes NHS Trust.

\section{MRI Technique}

A Bruker Medspec scanner, operating at $3 \mathrm{~T}$, was used to acquire gradient-echo echo planar $\mathrm{T} 2{ }^{*}$-weighted images depicting BOLD contrast. Images were acquired from 21 noncontiguous near axial planes: $\mathrm{TE}=27.5 \mathrm{~ms}$, flip angle $=66^{\circ}$, in-plane resolution $=3.1 \times 3.1 \mathrm{~mm}$, matrix size $64 \times 64$, field of view $20 \times 20 \mathrm{~cm}$, bandwidth $100 \mathrm{kHz}$. In total, 570 images were acquired for each session, six of which were discarded as 'dummy scans' to allow for T1 equilibration effects, leaving a total of 564 images per session. Volumes were acquired continuously every $1100 \mathrm{~ms}$. Each volume comprised 21 axial slices (each of $4 \mathrm{~mm}$ thickness, interslice gap $1 \mathrm{~mm}$; matrix size $128 \times 128$ ).

\section{Behavioral Tasks}

During scanning, subjects were required to complete a working memory task based on that used by D'Esposito et al (1999). A set of letters was presented for $2.5 \mathrm{~s}$, followed by an instruction cue ('FORWARD' or 'ALPHABET') for $1.5 \mathrm{~s}$. There was then a $7 \mathrm{~s}$ delay during which a fixation cross was presented on the screen, then a probe was displayed for $4 \mathrm{~s}$ during which the subject was required to respond with a 'yes' or a 'no' button press (see Figure 1). Total stimulus onset asynchrony was $15 \mathrm{~s}$. The letters in the initial stimulus were randomly generated consonants, excluding ' $\mathrm{Y}$ ', and were screened to exclude any common acronyms.

Subjects were scanned over two 10 min sessions (a break of $1 \mathrm{~min}$ was introduced to avoid fatigue). During each session, 10 trials of each type (low-load maintenance, low-load manipulation, high-load maintenance, high-load manipulation) were presented in randomized order. In the maintenance conditions, the cue was 'FORWARD', which indicated that subjects were required to remember the letters in the order that they were presented. The response cue was a question about the position of the letter (eg Was ' $\mathrm{N}$ ' 2nd?) and subjects were to give a 'yes' response if that letter had been presented in that position in the initial display, and a 'no' response if it had not. In the manipulation condition, the cue was 'ALPHABET', which indicated that subjects were required to mentally rearrange the letters into alphabetical order and to remember the letters in the new order. The response cue was the same as for the maintenance condition, that is, a question about the position of the letter (eg Was ' $N$ ' 2nd?), and subjects were to give a 'yes' response if that letter had that position alphabetically, and a 'no' response if it did not. For both the manipulation and maintenance condition, subjects were 


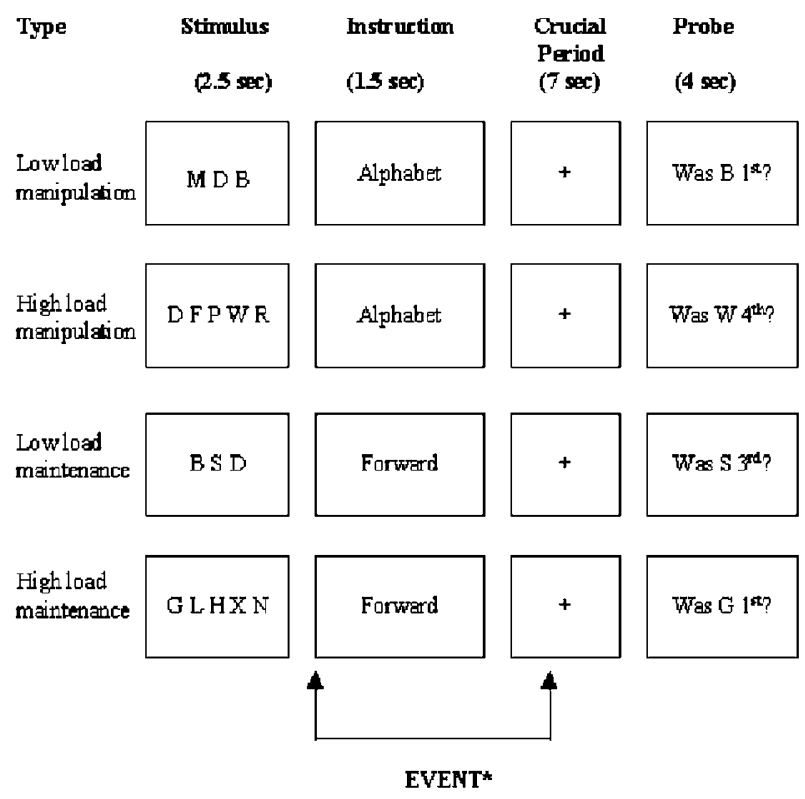

Figure I Experimental design. The two factors were load (high and low) and task (manipulation and maintenance) resulting in the four conditions shown. Following a $2.5 \mathrm{~s}$ presentation of the stimuli, subjects were given an instruction as to what was required. A $7 \mathrm{~s}$ delay period followed before the probe question appeared, requiring a yes/no (button push) response. The fMRI response to the instruction and delay period was modelled.

presented with three and five letters in the 'low'- and 'high'load conditions, respectively. Prior to scanning, participants were trained on this task with a brief practice involving eight sets of stimuli (two examples of each condition).

\section{Drug Administration Procedure}

The study was a double-blind, placebo-controlled, randomized, within-subjects comparison of targeted steady-state plasma levels of ketamine administered by continuous intravenous (i.v.) infusion. For safety reasons, the clinician administering the drug was aware of the study drug and dose being administered on each session. This clinician was not involved in test administration. Across their three visits subjects received saline placebo, ketamine infusion targeted at a plasma level of $50 \mathrm{ng} / \mathrm{ml}$ and ketamine infusion targeted $100 \mathrm{ng} / \mathrm{ml}$, the order of these infusions being randomized. Data for one subject for the $100 \mathrm{ng} / \mathrm{ml}$ condition and for one subject for the $50 \mathrm{ng} / \mathrm{ml}$ condition were lost due to technical problems.

Prior to testing, bilateral upper extremity i.v. catheters were inserted, one for ketamine infusion, the other for serial blood sampling for plasma ketamine levels. Racemic ketamine $(1 \mathrm{mg} / \mathrm{ml}$ solution) was administered by bolus and continuous infusion using a computerized pump (Graseby 3500, Graseby Medical Ltd UK). The pump was programmed (Anaetech Ltd UK) to deliver a rapid bolus dose $(<60 \mathrm{~s})$ followed by varying infusion rates in order to achieve constant estimated target plasma concentrations of 50 or $100 \mathrm{ng} / \mathrm{ml}$ within $2 \mathrm{~min}$, using pharmacokinetic parameters of a three-compartment model described by Domino et al (1982). Blinding of each subject and the investigator performing the clinical assessments was assured by use of the same infusion pump and pump adjustment schedule on all 3 study days (saline infusion on placebo day, ketamine infusions for the two intervention days) programmed by a single investigator not involved in clinical or cognitive assessment.

Simulations of plasma and effect-site (brain) concentrations of drugs with similar pharmacokinetics and effects (midazolam and propofol) suggests that plasma and effectsite concentrations equilibrate by $5 \mathrm{~min}$ following bolus administration (Gepts, 1998). Thus, behavioral testing began at least $5 \mathrm{~min}$ after the start of drug administration. The initial task was either the working memory task described above or a sustained attention task and this was followed by a long-term memory task. Data from the sustained attention and long-term memory task are not reported here.

Peripheral venous blood samples were drawn $10 \mathrm{~min}$ after testing started (15 min after beginning ketamine administration (Time 1)), and at the end of testing, approximately 65 min after beginning ketamine administration (Time 2). Blood samples were placed on ice, plasma obtained by centrifugation, and plasma samples stored at $-20^{\circ} \mathrm{C}$. The Ketamine levels were measured by gas chromatographymass spectrometry as previously described (Kharasch and Labroo, 1992).

\section{Data Analysis}

Data were analyzed with statistical parametric mapping (SPM2: www.fil.ion.ucl.ac.uk) implemented in Matlab (Mathworks Sherborn, MA).

Image preprocessing. Each volume was realigned to the first volume in the session and then realigned across sessions for each subject. Following estimation of movement parameters, this realignment was carried out with resampling of voxels using a sinc interpolation in space. A mean image was then normalized to standard stereotactic space (based on the Montreal Neurological Institute reference brain; Cocosco et al, 1997) using a 12 parameter affine transformation and nonlinear warping using basis functions (Ashburner and Friston, 1999). The parameters derived from this transformation were used to warp all volumes to the standard brain space. Finally, images were spatially smoothed using a Gaussian kernel $(8 \mathrm{~mm}$ full-width at halfmaximum).

Modelling BOLD responses. The time series in each session was high-pass filtered (average cutoff period $=120 \mathrm{~s}$ ). In order to account for serial autocorrelations, autoregression (first order) was applied. The event to be modelled was defined as an extended period from the presentation of the instruction to half way through the delay period (that is approximately $3.5 \mathrm{~s}$ ). This was based on the assumption that this was the time during which subjects would be continually performing the manipulation task, irrespective of load. It was expected that the high-load manipulation task would take the entire delay period, whereas the lowload manipulation task would be completed before the end of the delay period, after which subjects would simply be required to maintain the reordered stimulus set until the probe cue appeared. In all conditions, only correct responses were included in the analysis although incorrect 
responses were separately modelled in the design matrices.

The average hemodynamic responses to each event type were modelled using a canonical, synthetic hemodynamic response function (Friston et al, 1995). This function was used as a covariate in a general linear model in order to generate a parameter estimate for each voxel for each of the four event types. The parameter estimate, derived from the mean least-squares fit of the model to the data, reflects the strength of covariance between the collected time series and the canonical response function for a given condition. Responses to different event types were then compared within subjects by setting up pairwise contrasts between the parameter estimates for these events in order to generate a contrast image for each contrast for each subject. These contrast images were taken through to a group analysis in which intersubject variability was treated as a random effect. The $t$-values were transformed to unit normal $Z$ distribution to create a statistical parametric map for each of the planned contrasts, which are described below:

(a) Preliminary analyses - main effects of task manipulations under placebo and ketamine separately: We initially carried out a series of analyses identifying the main effects of the two factors for the placebo condition only and for the combined ketamine conditions (lower and higher dose). These comprised:

1. Simple main effects of task type (manipulation of information $v s$ maintenance of information and vice versa).

2. Simple main effects of load represented by the number of letters required to be maintained or manipulated by the subject.

Statistical thresholding: Each of these contrasts was thresholded using a control for the False Discovery Rate (FDR). This statistical thresholding was chosen in view of criticisms that have been levelled at the use of a bonferronitype correction for multiple comparisons that has frequently been used in functional neuroimaging studies (Genovese et al, 2002). Specifically, the level of type II error with a bonferroni-type correction may be unacceptably high. FDR control obviates the multiple comparisons problem by controlling for the proportion of falsely rejected null hypotheses among all rejected null hypotheses. Thus for each region identified using FDR control, we can specify the likelihood that this was a type I error. We chose a FDR threshold of $p<0.05$.

(b) Analyses of drug effects: Drug effects upon taskdependent activations were characterized using two approaches. In the first, we identified the main effect of drug (all drug $v s$ placebo) using a $t$-test comparing activations on and off ketamine (irrespective of dose). In the second, we identified linear dose-dependent effects using a one-way ANOVA model (with three levels of taskdependent activation: higher dose ketamine $v s$ lower dose ketamine $v s$ placebo).

In order to unpack a complex series of drug by task interactions, we applied each of these two models (main effects and linear dose-dependent effects) to pairwise comparisons of the four experimental tasks in the following way:

(i) Effects of load in the maintenance task (high-load maintenance $v s$ low-load maintenance).

(ii) Effects of load in the manipulation task (high-load manipulation vs low-load manipulation).

(iii) Effects of manipulation in association with low load (low-load manipulation vs low-load maintenance).

(iv) Effects of manipulation in association with high load (high-load manipulation $v s$ high-load maintenance).

(v) The interaction between load and manipulation.

Statistical thresholding: Drug effects are likely to be subtle since they reflect modulations of task-related activation. We used a two-stage approach to their identification, aimed at maximizing our sensitivity to the drug effects without inflating the risk of false positives to unacceptable levels. In the first stage, we identified the brain regions of interest by creating masks of the main effects of different tasks comparisons (placebo and drug combined). These masks were not intended to be the basis for statistical inference and were therefore thresholded leniently $(p<0.005$, uncorrected for multiple comparisons). The subsequent analyses of interest (drug by task interactions) were confined to the regions identified by these masks and were thresholded using FDR $(p<0.05)$ taking into account the reduced volume of interest. Thus, for example, the analysis of drug-load interactions was confined to those areas in which a load effect was seen across placebo and drug conditions. The same was carried out for the manipulation $v s$ maintenance comparisons. This 'masking' procedure, as well as reducing the multiple comparisons problem, focuses analyses upon task-related systems.

\section{RESULTS}

\section{Plasma Levels}

The mean plasma levels are presented in Table 1. At Time 2 (predicted steady state), measurement closely approximated the levels predicted by the pharmacokinetic model with a mean of $47.7 \mathrm{ng} / \mathrm{ml}$ in the $50 \mathrm{ng} / \mathrm{ml}$ dose condition and $102.3 \mathrm{ng} / \mathrm{ml}$ in the $100 \mathrm{ng} / \mathrm{ml}$ dose condition.

Table I Means and Standard Deviations (ng/ml) for Plasma Levels of Ketamine at 15 min Postinfusion (Time I) and $65 \mathrm{~min}$ Postinfusion (Time 2) for the $50 \mathrm{ng} / \mathrm{ml}$ Ketamine and $100 \mathrm{ng} / \mathrm{ml}$ Conditions

\begin{tabular}{lccc}
\hline & Mean & Standard deviation & Range \\
\hline Time I & & & \\
$50 \mathrm{ng} / \mathrm{ml}$ & 34.7 & 9.1 & $21.4-50.5$ \\
$100 \mathrm{ng} / \mathrm{ml}$ & 65 & 12.6 & $43.9-80.3$ \\
& & & \\
Time 2 & & 11.6 & $34.1-66.4$ \\
$50 \mathrm{ng} / \mathrm{ml}$ & 47.7 & 21.5 & $70.8-123.4$ \\
$100 \mathrm{ng} / \mathrm{ml}$ & 102.35 & & \\
\hline
\end{tabular}


The plasma levels at Time 1 were substantially lower than those predicted by the model with a mean of $34.7 \mathrm{ng} / \mathrm{ml}$ in the $50 \mathrm{ng} / \mathrm{ml}$ dose condition and a mean of 65.0 in the $100 \mathrm{ng} / \mathrm{ml}$ dose condition.

\section{Behavioural Results}

The results of the behavioral task are presented in Table 2 . A 2 (low load, high load) $\times 2$ (maintenance, manipulation) $\times 3$ (placebo, $50 \mathrm{ng} / \mathrm{ml}, 100 \mathrm{ng} / \mathrm{ml}$ ) repeated measures ANOVA for reaction time effects revealed a significant main effect of load, $\mathrm{F}(1,10)=116.21, p<0.05$ and task type, $\mathrm{F}(1,10)=6.27, p<0.05$, with a significantly slower reaction time in the high-load compared to low-load conditions and a significantly slower reaction time for the manipulation compared to the maintenance conditions. There was no significant main effect of drug, $\mathrm{F}(2,20)=0.18, p=0.84$, and no significant interactions between drug and load, $\mathrm{F}(2,20)=0.58, p=0.57$, between drug and task, $\mathrm{F}(2,20)=1.71, p=0.20$, or between drug, load, and task, $\mathrm{F}(2,20)=2.54, p=0.10$. A $2 \times 2 \times 3$ repeated measures ANOVA for percent of correct responses revealed a significant main effect of load, $\mathrm{F}(1,10)=50.17, p<0.05$, and task type, $\mathrm{F}(1,10)=11.65, p<0.05$, with significantly more correct in the low-load compared to the high-load conditions and significantly more correct in the manipulation compared to the maintenance conditions. There was no significant main effect of drug, $\mathrm{F}(2,20)=0.02, p=0.98$, and no significant interactions between drug and load, $\mathrm{F}(2,20)=1.61, \quad p=0.22$, between drug and task, $\mathrm{F}(2,20)=0.55, p=0.58$, or between drug, load, and task, $\mathrm{F}(2,20)=0.15, p=0.86$.

Thus, the task manipulation produced an effect on performance in terms of response times and error rates

Table 2 Means and Standard Deviations of the Reaction Time and Percent of Correct Responses in the Low-Load Maintenance, Low-Load Manipulation, High-Load Maintenance, and High-Load Manipulation Conditions

\begin{tabular}{|c|c|c|c|}
\hline Measure & $\begin{array}{l}\text { Placebo mean } \\
\text { (SD) }\end{array}$ & $\begin{array}{c}50 \mathrm{ng} / \mathrm{ml} \\
\text { ketamine mean } \\
\text { (SD) }\end{array}$ & $\begin{array}{c}100 \mathrm{ng} / \mathrm{ml} \\
\text { ketamine mean } \\
\text { (SD) }\end{array}$ \\
\hline \multicolumn{4}{|c|}{ Low-load maintenance } \\
\hline Reaction time & | $898.82(228.35)$ & | 883.00 (394.77) & $1942.34(332.84)$ \\
\hline Percent correct & 91.25 (9.32) & 94.55 (8.79) & $94.58(9.88)$ \\
\hline \multicolumn{4}{|c|}{ Low-load manipulation } \\
\hline Reaction time & | $897.68(284.14)$ & | $87 \mid .75$ (408.45) & 1964.23 (287.46) \\
\hline Percent correct & $87.92(12.15)$ & $90.45(14.40)$ & $87.50(13.90)$ \\
\hline \multicolumn{4}{|c|}{ High-load maintenance } \\
\hline Reaction time & $2264.97(319.65)$ & $2223.83(355.17)$ & $2251.75(231.04)$ \\
\hline Percent correct & $79.17(15.20)$ & 79.09 (16.25) & $78.33(16.56)$ \\
\hline \multicolumn{4}{|c|}{ High-load manipulation } \\
\hline Reaction time & $2229.48(373.16)$ & $2432.78(432.17)$ & $2345.31(408.49)$ \\
\hline Percent correct & $71.25(15.39)$ & $68.18(16.01)$ & $65.00(21.11)$ \\
\hline
\end{tabular}

but there was no measurable effect of drug on performance of any of the tasks.

\section{Imaging Results}

(a) Preliminary analyses: task effects under placebo and ketmain separately

\section{(1) Effects of tasks under placebo condition}

Manipulation vs maintenance. The contrast between manipulation and maintenance conditions collapsed across load level produced bilateral activation of the dorsolateral prefrontal region, ventrolateral prefrontal regions, parietal cortex, the anterior cingulate gyrus, the globus pallidus, the caudate nucleus, the putamen, and thalamic regions (see Table 3 and Figure 2a). The reverse contrast between maintenance and manipulation conditions showed bilateral activation of the temporal lobe (see Table 3).

High vs low load: The contrast between high and low load, collapsed across task type, produced bilateral activation of the dorsolateral prefrontal region, parietal areas, and

Table 3 Regions of Activation within the Placebo Condition for the Manipulation Compared to Maintenance Contrast (Manip vs Maint), and the Low-Load Manipulation Compared to Low-Load Maintenance Contrast (Low Manip vs Low Maint)

\begin{tabular}{|c|c|c|c|c|}
\hline Region activated & $x^{a}$ & $Y^{a}$ & $z^{a}$ & Z-score \\
\hline \multicolumn{5}{|l|}{ Manipulation vs maintenance } \\
\hline Right dorsolateral prefrontal & 48 & 4 & 28 & $4.44 * *$ \\
\hline Left dorsolateral prefrontal & -42 & 8 & 28 & $4.25 * *$ \\
\hline Right ventrolateral prefrontal & 40 & 18 & 10 & 4.30*** \\
\hline Left ventrolateral prefrontal & -34 & 28 & 4 & $4.00 * *$ \\
\hline Right parietal & 42 & -48 & 38 & $4.82 * *$ \\
\hline Left parietal & -44 & -42 & 38 & $4.76 * *$ \\
\hline Anterior cingulate gyrus & -4 & 22 & 46 & $3.95 * *$ \\
\hline Globus pallidus & 16 & -8 & 0 & $3.90 * *$ \\
\hline Caudate nucleus & 32 & 20 & -8 & $3.39 * *$ \\
\hline Putamen & -16 & -12 & 10 & $3.29 * *$ \\
\hline Thalamus & -8 & 4 & 8 & $3.26 * *$ \\
\hline \multicolumn{5}{|l|}{ Maintenance vs manipulation } \\
\hline Left temporal & -50 & -28 & 18 & 4.07 \\
\hline \multicolumn{5}{|l|}{ High load vs low load } \\
\hline Right dorsolateral prefrontal & 50 & 24 & 32 & $3.87 * *$ \\
\hline Left dorsolateral prefrontal & -36 & 6 & 34 & $5.28 * *$ \\
\hline Right parietal & 34 & -78 & 40 & $4.25 * *$ \\
\hline Left parietal & -36 & -74 & 34 & $3.78 * *$ \\
\hline Anterior cingulate gyrus & -14 & 18 & 52 & $4.71 * * *$ \\
\hline Thalamus & 8 & -4 & 0 & $4.11 * *$ \\
\hline
\end{tabular}

${ }^{a} X, Y$, and $Z$ are coordinates in a standard stereotactic space in which positive values refer to regions right of $(X)$, anterior to $(Y)$, and superior to $(Z)$ the anterior commissure.

*** $p<0.05$ (FDR). 
a

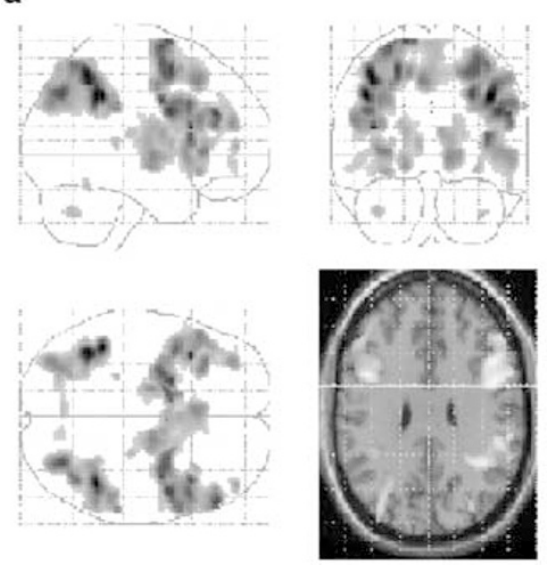

b

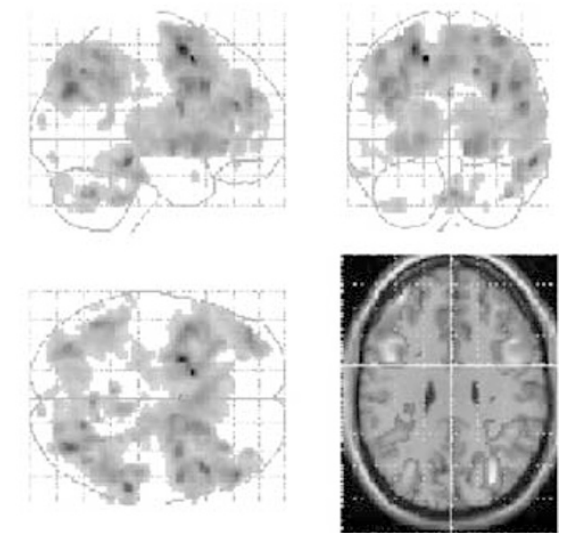

Figure 2 (a) SPM showing the contrast between manipulation and maintenance conditions collapsed across load for the placebo condition. 'Glass brain' images are presented showing coronal, sagittal, and axial views. In order to identify the localization of main frontal and parietal activations, these are mapped onto a standard anatomical image in the same space at $30 \mathrm{~mm}$ above the ac-pc line. Images are presented at $p<0.05$ (FDR corrected). Clusters less than I0 contiguous voxels have been excluded. (b) SPM showing the contrast between manipulation and maintenance conditions collapsed across load for the ketamine conditions. Images are prepared and represented as described above.

the anterior cingulate gyrus for the high-load condition compared to the low-load condition (see Table 3 and Figure 3a). The reverse contrast (low load vs high load) did not produce any activation surviving our chosen threshold.

Interactions: No interactions between process and load were seen at the set threshold.

\section{(2) Effects of tasks under Ketamine conditions}

Manipulation vs maintenance. The contrast between manipulation and maintenance conditions collapsed across load level produced bilateral activation of the dorsolateral prefrontal region, ventrolateral prefrontal regions, parietal cortex, the anterior cingulate gyrus, the caudate nucleus, and the thalamus (see Table 4 and Figure $2 \mathrm{~b}$ ). The reverse contrast between maintenance and manipulation conditions showed bilateral activation of the temporal lobe (see Table 4).

High vs low load: The contrast between high and low load, collapsed across task type, produced bilateral activation of the dorsolateral prefrontal region, parietal areas, and the anterior cingulate gyrus for the high-load condition compared to the low-load condition (see Table 4 and Figure $3 \mathrm{~b}$ ). The reverse contrast (low load vs high load) did not produce any activation surviving our chosen threshold.

\section{(b) Drug effects}

The effects of ketamine were calculated using a small volume correction based on a mask produced by the comparisons for the placebo and ketamine conditions combined, that is, the combination of the above comparisons. For the manipulation vs maintenance comparisons, the mask comprised bilateral dorsolateral and ventrolateral prefrontal regions, bilateral parietal regions, the anterior
Table 4 Regions of Activation within the Combined Higher and Lower Dose Ketamine Conditions for the Manipulation Compared to Maintenance Contrast (Manip vs Maint), and the Low-Load Manipulation Compared to Low-Load Maintenance Contrast (Low Manip vs Low Maint)

\begin{tabular}{lrrrl}
\hline Regions activated & $\boldsymbol{X}^{\mathbf{a}}$ & $\boldsymbol{Y}^{\mathbf{a}}$ & $\mathbf{Z}^{\mathbf{a}}$ & Z-score \\
\hline Manipulation vs maintenance & & & & \\
Right dorsolateral prefrontal & 44 & 16 & 36 & $\left.4.6\right|^{* *}$ \\
Left dorsolateral prefrontal & -44 & 6 & 34 & $4.46 * *$ \\
Right ventrolateral prefrontal & 46 & 22 & 18 & $3.84 * *$ \\
Left ventrolateral prefrontal & -34 & 28 & -6 & $3.90^{* * *}$ \\
Right parietal & 46 & -50 & 42 & 4.1 * $^{*} *$ \\
Left parietal & -46 & -44 & 38 & $3.99 * *$ \\
Anterior cingulate gyrus & -4 & 18 & 46 & $3.97 * *$ \\
Caudate nucleus & 14 & -16 & 0 & $4.25 * *$ \\
Thalamus & 12 & 28 & 12 & $2.67 * *$ \\
& & & & \\
High load vs low load & & & & \\
Right dorsolateral prefrontal & 46 & 26 & 30 & 2.95 \\
Left dorsolateral prefrontal & -24 & 4 & 48 & 3.9 \\
Right parietal & 26 & -58 & 44 & 2.94 \\
Left parietal & -28 & -54 & 26 & 2.92 \\
Anterior cingulate gyrus & -10 & 22 & 38 & 3.94 \\
\hline
\end{tabular}

${ }^{a} X, Y$, and $Z$ are coordinates in a standard stereotactic space in which positive values refer to regions right of $(X)$, anterior to $(Y)$, and superior to $(Z)$ the anterior commissure.

*** $p<0.05$ (FDR).

cingulate, thalamic regions, the caudate nucleus, the putamen, and the globus pallidus. For the load contrasts, the mask comprised bilateral dorsolateral and ventrolateral prefrontal regions, bilateral parietal regions, and the anterior cingulate. 
a

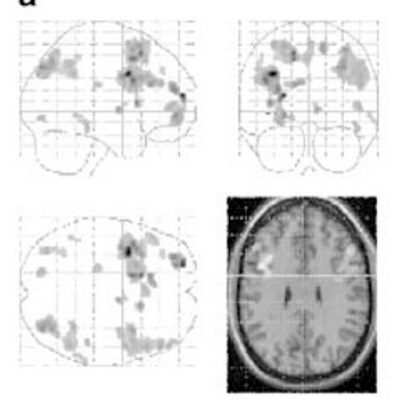

b

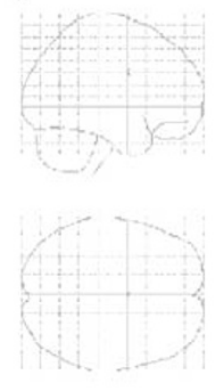

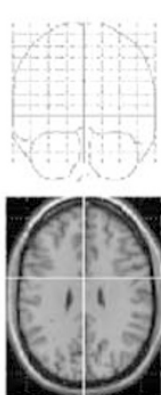

Figure 3 (a) SPM showing the contrast between high load and low load collapsed across task type (manipulation and maintenance) for the placebo condition. (b) SPM showing the contrast between high load and low load collapsed across task type (manipulation and maintenance) for the ketamine conditions. Images are prepared and represented as for Figure 2.

\section{Manipulation $v s$ Maintenance}

(1) Main effect of drug

Manipulation vs maintenance. The contrast between ketamine and placebo for the manipulation $v s$ maintenance comparison showed an augmentation associated with ketamine administration in the entire system that was associated with task performance: namely, bilateral dorsolateral prefrontal regions, ventrolateral prefrontal regions, parietal areas, and the anterior cingulate gyrus (see Table 5 and Figure 4a).

High-load manipulation vs maintenance: The contrast between ketamine and placebo for the high-load manipulation $v s$ high-load maintenance comparison failed to show any significant difference at the chosen threshold (see Figure $4 b)$.

Low-load manipulation vs maintenance: The contrast between ketamine and placebo for the low-load manipulation $v s$ low-load maintenance comparison showed an augmentation associated with ketamine administration in the following regions: bilateral dorsolateral prefrontal regions, right ventrolateral prefrontal cortex, parietal areas, and the anterior cingulate gyrus (see Table 5 and Figure 4c).

\section{(2) Dose-dependent effect of drug}

Manipulation vs maintenance. A one-way repeated measures ANOVA with drug condition as the independent variable (placebo, lower dose ketamine, higher dose ketamine) failed to show any significant effect of drug for the comparison between manipulation and maintenance processes at the chosen threshold.

High-load manipulation vs maintenance: A one-way repeated measures ANOVA with drug condition as the independent variable (placebo, lower dose ketamine, higher dose ketamine) failed to show any significant effect of drug for the comparison between manipulation and maintenance processes within the high-load condition at the chosen threshold.
Table 5 Relative Increases in Activation for the Manipulation Compared to Maintenance Contrast for Combined Lower and Higher dose Ketamine Conditions Compared with Placebo (Manip vs Maint), and the Low-Load Manipulation Compared to Low-Load Maintenance Contrast for Combined Lower and Higher Dose Ketamine Conditions Compared with Placebo (Low Manip vs Low Maint)

\begin{tabular}{|c|c|c|c|c|}
\hline Regions activated & $x^{a}$ & $Y^{\mathbf{a}}$ & $z^{a}$ & Z-score \\
\hline \multicolumn{5}{|l|}{ Manipulation vs maintenance } \\
\hline Right dorsolateral prefrontal & 50 & 10 & 42 & $4.20 * *$ \\
\hline Left dorsolateral prefrontal & -46 & 4 & 32 & $4.12 * *$ \\
\hline Right ventrolateral prefrontal & 44 & 28 & 16 & 4.10** \\
\hline Left ventrolateral prefrontal & -32 & 20 & -2 & $4.13 * *$ \\
\hline Right parietal & 32 & -74 & 48 & $3.85 * *$ \\
\hline Left parietal & -38 & -60 & 42 & $3.03 * *$ \\
\hline Anterior cingulate gyrus & -18 & 8 & 52 & $3.46 * *$ \\
\hline Putamen & 16 & 12 & -6 & $3.64 * *$ \\
\hline \multicolumn{5}{|c|}{ Low manipulation vs low maintenance } \\
\hline Right dorsolateral prefrontal & 52 & 34 & 28 & $3.36 * *$ \\
\hline Left dorsolateral prefrontal & -46 & 14 & 46 & $3.93 * *$ \\
\hline Right ventrolateral prefrontal & 44 & 28 & 16 & $3.15 * *$ \\
\hline Right parietal & 30 & -62 & 42 & $4.11 * *$ \\
\hline Left parietal & -32 & -56 & 44 & $3.49 * *$ \\
\hline Anterior cingulate gyrus & -18 & 10 & 48 & $3.81 * * *$ \\
\hline Caudate nucleus & -16 & 16 & 14 & $3.96 * *$ \\
\hline Putamen & 20 & 20 & 2 & $2.04 * *$ \\
\hline
\end{tabular}

${ }^{a} X, Y$, and $Z$ are coordinates in a standard stereotactic space in which positive values refer to regions right of $(X)$, anterior to $(Y)$, and superior to $(Z)$ the anterior commissure.

*** $p<0.05$ (FDR).

Low-load manipulation vs maintenance: A one-way repeated measures ANOVA with drug condition as the independent variable (placebo, lower dose ketamine, higher dose ketamine) failed to show any significant effect of drug for the comparison between manipulation and maintenance processes within the low-load condition at the chosen threshold.

\section{Load Effects}

(1) Main effect of drug

High load vs low load. The contrast between ketamine and placebo for the high-load $v s$ low-load comparison failed to show any significant effect of drug at the chosen threshold.

Load effects within the manipulation condition: The contrast between ketamine and placebo for the high-load manipulation $v s$ low-load manipulation comparison failed to show any significant effect of drug at the chosen threshold.

Load effects within the maintenance condition: The contrast between ketamine and placebo for the high-load maintenance $v s$ low-load maintenance comparison failed 
a
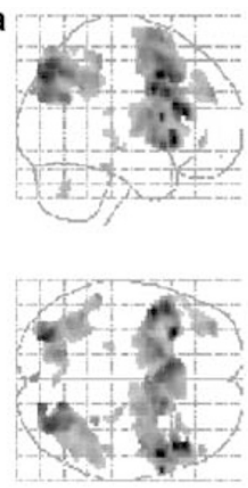

b

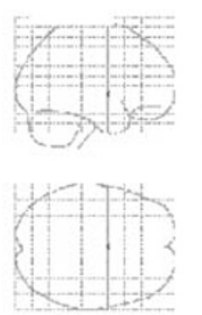

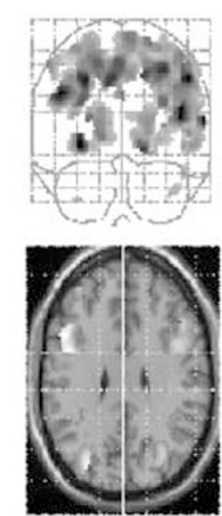

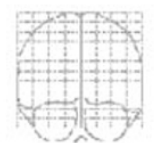

C

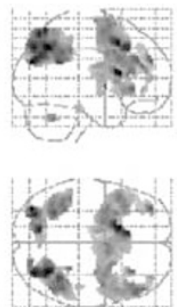

As a supplementary analysis, the above contrasts were run at a reduced (uncorrected) threshold $(p<0.001)$. Again no effects of drug on load-related activations were seen.

Exploring load effects using a model taking into account latency of the hemodynamic response: Having found no evidence of a drug effect on the load manipulation, the question arises as to whether this might result from an inadequacy of the model used: we initially modelled events using the canonical hemodynamic response function (HRF) in SPM. It is possible, therefore that if ketamine has an effect upon the onset of the BOLD response, then our model might be suboptimal with respect to detecting condition-specific drug effects. In order to check this, we subsequently set up a model that included the first temporal derivative of the HRF. We ran the high- $v s$ low-load contrasts using this extended model and again, found no effect of the drug in the regions of interest (even at a muchreduced threshold of $p<0.01$, uncorrected). Thus, a loadby-drug interaction is not seen even when we allow for differential effects on the HRF onset.

\section{Interaction (Load by Task by Drug)}

Fig (a) SPM showing the drug by task interaction. This figure identifies regions in which the manipulation vs maintenance contrast (collapsed across load) is greater under ketamine (collapsed across doses) than placebo. Glass brain images were prepared and thresholded as for Figure 2. An axial slice at $30 \mathrm{~mm}$ above ac-pc line is presented with activations superimposed. (b) Drug by task interaction prepared as for (c) but showing the ketamine-related increase for high-load conditions only. (c) Drug by task interaction prepared as for (c) Figure 4c but showing the ketamine-related increase for low-load conditions only. NB. All images (Figure 4) are presented at $p<0.05$ (FDR) and with a threshold level of 10 voxels. All images presented are constrained by masks that were generated from the contrast between manipulation and maintenance conditions collapsed across load for all three drug conditions (placebo, lower dose, and higher dose). Thresholds defining this mask were set at $p<0.005$, uncorrected for multiple comparisons.

to show any significant effect of drug at the chosen threshold.

\section{(2) Dose-dependent effect of drug}

High load vs low load. A one-way repeated measures ANOVA with drug condition as the independent variable (placebo, lower dose ketamine, higher dose ketamine) failed to show any significant effect of drug at the chosen threshold.

Load effects within the manipulation condition: A oneway repeated measures ANOVA with drug condition as the independent variable (placebo, lower dose ketamine, higher dose ketamine) failed to show any significant effect of drug at the chosen threshold.

Load effects within the maintenance condition: A oneway repeated measures ANOVA with drug condition as the independent variable (placebo, lower dose ketamine, higher dose ketamine) failed to show any significant effect of drug for the comparison between high-load and lowload processes within the maintenance condition at the chosen threshold.

The analyses of the effects of the interaction between task type and load failed to show any significant difference between drug conditions and placebo. A subsequent analysis at much lower threshold ( $p<0.01$, uncorrected) showed tentative evidence for a three-way interaction in frontal cortex. We refer briefly to this in the discussion section but are cautious in drawing any conclusions.

\section{Summary of Results}

Both on and off ketamine, the expected activations were seen in association with the more demanding working memory tasks. Thus, the requirement to reorganize material was associated with greater fronto-parietal activation than a working memory task requiring merely the maintenance of information. Furthermore, an increase in load (the number of stimuli held in working memory) produced increased activation in the same system for both the manipulation and the maintenance tasks.

The effects of ketamine are seen only in relation to manipulation processes. Thus, comparing manipulation to maintenance tasks (collapsed across load), there is an augmentation of activation in fronto-parietal regions on ketamine (see Figure 4a). This effect was not observed (at the chosen threshold) with increasing load. Exploring the effect of manipulation within each load separately (see Figures $4 \mathrm{~b}$ and c) enables us to identify a specific effect of ketamine in association with an augmentation of taskrelated activation within the manipulation task under lowload conditions. Under high-load conditions, manipulation is associated with high levels of activation in this system both under placebo and ketamine. That is, it seems that manipulation processes require a high degree of activation under ketamine even under low-load conditions, but that, on placebo, the same level of activation is only reached under high-load conditions. 


\section{DISCUSSION}

Under ketamine administration, compared to placebo, performance of a task requiring manipulation of verbal information is associated with greater activation in bilateral dorsolateral prefrontal regions, bilateral ventrolateral areas, bilateral parietal cortices, the anterior cingulate, the putamen, and the caudate nucleus. These comprise the network of areas associated with the manipulation of verbal information in the placebo condition. On closer inspection, it appears that this increase in activation is driven largely by the low-load manipulation condition. This shows that, even when the task is performed under the easiest conditions, when there are no overt indications of behavioral impairments, it is possible to identify process-specific effects of ketamine. It also suggests that the effects of ketamine are not simply a result of increasing the difficulty of the task.

So, consistent with our prediction, we have shown a taskspecific effect of ketamine on brain activation even in the absence of measurable behavioral impairment. This is noteworthy for two reasons: first, it indicates a potentially complementary role for psychopharmaocological fMRI studies, since, evidently, the regional BOLD response distinguishes between drug and placebo conditions even when performance does not. Second, the task specificity of the fMRI findings is consistent with that seen in our previous study in which the emphasis was upon an incremental increase in task difficulty in order to establish the pattern of behavioral impairments produced by the drug. We discuss these points below.

\section{fMRI May Detect Physiological Changes in the Absence of Behavioral Impairment}

An important facet of our study was that subjects' behavioral performance did not show any signs of deterioration as a result of ketamine administration. We ensured this through modification of the task design and through exploitation of the experimental flexibility allowed by event-related fMRI, which allowed us to exclude from the analysis all trials associated with incorrect responses. One important reason for this feature of our design and analysis is that, when interpreting brain activations across drug and placebo conditions, performance must be taken into account. Activations will reflect the results of task performance as well as the cognitive processes that engender it. If two groups differ in terms of task success, then differences in activation may reflect this rather than differences in the cognitive processes of interest.

It is worth considering the implications of our observation of drug-induced modulations of the BOLD response in the face of apparently preserved task performance. We suggest that this indicates the potentially complementary role of psychopharmacological fMRI studies due to their sensitivity to cognitive changes that may not be seen behaviorally. An absence of a behavioral effect could arise because of a lack of statistical power. We must concede that, with the low number of subjects in the current study, this should be taken into account. That is, it may be the case that ketamine does have an effect on performance of our experimental tasks and that we would have seen this with more trials in more subjects. If this were so (although our previous findings suggest that ketamine does not impair performance at these levels of working memory load, but only when the demands increase (Honey et al, 2003)), then it is noteworthy that the BOLD measurements were sensitive to the drug's effects. Alternatively, one can envisage situations in which a drug produces cognitive effects that could not be seen using standard behavioral measures. This seems especially likely with frontally mediated processes in which performance might be maintained through a variety of strategies between which subjects may switch without any overt changes (see Burgess, 1997 for a discussion). In such cases, where cognitive change is behaviorally silent, functional neuroimaging may provide important additional information, indicating whether apparently unchanged behavioral performance under the drug is truly a reflection of unchanged cognitive processing.

\section{Task-Specificity of the Findings}

Previous studies examining the effect of ketamine on blood flow while participants are at rest have found increased activity in cingulate and prefrontal regions (Breier et al, 1997; Holcomb et al, 2001; Lahti et al, 1995; Vollenweider et al, 1997b). This is consistent with our results showing changes predominantly in these regions.

The task-specificity of drug-related activation changes agrees with and complements our prior behavioral findings (Honey et al, 2003). Both studies have shown an effect of ketamine upon the executive control of working memory but not upon simple maintenance of material. It is important to note that this effect was shown at very low doses of ketamine. At slightly higher doses, previous studies have shown an effect of ketamine on short-term maintenance of verbal information (Krystal et al, 1994). We differentiate manipulation from maintenance in keeping with the majority of functional neuroimaging studies of working memory (Fletcher and Henson, 2001). Manipulation refers to the processes that go beyond simple maintenance of information and involve updating and reordering this information. Further, such a task presumably demands the repeated monitoring of the results of these manipulations. These processes may be engaged in a number of tasks, such as the n-back task (Braver et al, 1997; Jonides et al, 1997). We chose a modification of the task of D'Esposito and colleagues since this allows us to vary manipulation and load separately. There are, however, a number of drawbacks to our study design. For example, while it is possible to have maintenance occurring in the absence of manipulation, the reverse is not true. Furthermore, we were unable to dissociate fully brain responses to the working memory demands of the task from those associated with decision making and responses which might vary across conditions. Finally, we must ultimately exercise caution in interpreting the task specificity of our findings since we must express this, in part, with reference to a null finding: the absence of an effect of ketamine on BOLD responses to increasing load. Like any null finding, this demands caution. We note, although, that no effect of ketamine was seen on this experimental factor even at lower, uncorrected thresholds, and when we used an expanded model taking into account possible modulations of the shape of the BOLD response. 
One further point should be made with respect to the apparent task specificity of our findings. There are a number of studies indicating that ketamine impairs memory processes when there is no explicit engagement of higher order control processes such as those required in the manipulation task used here (eg Ghoneim et al, 1985; Oye et al, 1992; Krystal et al, 1994). We do not view our findings as incompatible with these. First, we have used lower doses of the drug and, second, these studies have concentrated upon ketamine's effects upon episodic memory, while our study has solely engaged short-term memory.

The mechanism by which ketamine may lead to taskrelated hyperactivity can only be speculated upon. Glutamate has widespread effects upon other neurotransmitter systems, including dopaminergic, monoaminergic, and GABAergic (Tamminga, 1998, 1999; Vollenweider et al, 1997a). Thus, for example, NMDA blockade may attenuate the inhibitory GABAergic system leading to an increase in cortical activity due to the consequent increase in monoaminergic (Vollenweider et al, 1997a) or dopaminergic (Tsai and Coyle, 2002; Smith et al, 1998; Tamminga, 1998) activity. Alternatively, particularly at lower doses, such as those used here, ketamine may produce an enhancement of glutamatergic activity by increasing levels of glutamate, which act on non-NMDA receptors (Moghaddam et al, 1997; Kegeles et al, 2000) activity. While we may speculate upon the mechanism for a general increase in cortical activity, we must also consider the selective, task-dependent hyperactivity that we observed. Olney and Farber (1995) argue that as well as modulating inhibitory control of the cortex, glutamate is important in providing negative feedback which allows the cortex to monitor and restrain its discharge activity. Such monitoring demands are a key component of the manipulation conditions (Fletcher and Henson, 2001) and may explain why the drug effects were prominent in the manipulation task.

There is a further fundamental question about the augmentation of brain activation produced by ketamine. Our results alone offer no clues as to whether it reflects impairment or an enhancement of glutamatergic processing. The low doses used here raise the possibility that we are observing, primarily, the effects of a non-NMDA receptor-based glutamatergic enhancement (Moghaddam et al, 1997) and the increase in cortical activity could reflect enhanced function. Alternatively, the augmented activation may reflect an impairment or inefficiency in processing. This would be compatible with the finding from our behavioral study that, at the same doses, a performance impairment is seen when task demands are increased (Honey et al, 2003). This would predict that, while the fronto-parietal system is heavily engaged under ketamine in the low-load manipulation condition, activation would fall away as demands became greater. Such a shift in the loadactivation profile would be consistent with an inverted $\mathrm{U}$ shape relationship between dorsolateral activation and working memory performance, that is, activation increases until a threshold is met after which behavioral performance and brain activity decline (Callicott et al, 1999). With respect to our study, we would therefore predict load by manipulation by drug interaction (ie a reduction in the drug augmentation of manipulation activity when moving from low to high load). This interaction was only seen at a reduced threshold $(p<0.01$, uncorrected). The prediction would be that, with increasing load, performance and frontal activation would fall away. However, we may only speculate upon this.

Two other points are worth discussing briefly. First, the dose dependence of our findings. In the comparison of manipulation and maintenance tasks, the most statistically compelling effect of the drug emerged from a comparison of all drug $v s$ placebo. This suggests that the two doses used produced a similar effect on the BOLD response. This apparent dose independence perhaps arises from the small dose differences in our protocol. It is an interesting observation viewed in light of the behavioral study in which the higher, but not the lower dose, produced impairment as task demands grew. Together, the two studies suggest that the behavioral measure is sensitive to the difference between the two doses but not to any subtle effects of the lower one, whereas the neuroimaging measure detects a subbehavioral effect of the lower dose but not a dose-related effect.

Second, we must acknowledge that a concern with these results is that the plasma levels failed to reach predicted levels after $5 \mathrm{~min}$ of infusion. Participants started performing the task at this time, that is, predicted plasma levels probably had not been reached during the start of this task. However, this reduction in plasma levels would have diminished any cognitive or physiological effect. Consequently, it is unlikely that our results exaggerate the effect, rather, they probably underestimate the effect of ketamine.

In summary, the results of this study show that the administration of ketamine at low doses leads to hyperactivity in the entire network required to perform a task involving the manipulation of verbal information when the number of letters to be manipulated is low. The relationship of these observations to schizophrenia warrants further analysis of the same tasks performed in patients. We suggest that, our observations of extensive neurophysiological disruption, even in the absence of florid psychosis may begin to provide a useful basis for consideration of the early cognitive changes associated with the condition.

\section{ACKNOWLEDGEMENTS}

PCF, RAEH, and GDH are supported by the Wellcome Trust. We are very grateful to Gloria Gee and others at the Wolfson Brain Imaging Centre. In addition, Irene Tracey and her team at Oxford University were extremely helpful and generous in their advice.

\section{REFERENCES}

Adler CM, Goldberg TE, Malhotra AK, Pickar D, Breier A (1998). Effects of ketamine on thought disorder, working memory, and semantic memory in healthy volunteers. Biol Psychiatry 43: 811816.

Ashburner J, Friston KJ (1999). Nonlinear spatial normalization using basis functions. Hum Brain Mapp 7: 254-266.

Baddeley AD (1986). Working Memory. Clarendon Press: Oxford, UK.

Baddeley AD, Hitch GJ (1994). Developments in the concept of working memory. Neuropsychology 8: 485-493. 
Braver TS, Cohen JD, Nystrom LE, Jonides J, Smith EE, Noll DC (1997). A parametric study of prefrontal cortex involvement in human working memory. Neuroimage 5: 49-62.

Breier A, Malhotra AK, Pinals DA, Weisenfeld NI, Pickar D (1997). Association of ketamine-induced psychosis with focal activation of the prefrontal cortex in healthy volunteers. Am J Psychiatry 154: 805-811.

Bressi S, Miele L, Bressi C, Astori S, Gimosti E, Linciano AD et al (1996). Deficit of central executive component of working memory in schizophrenia. $N$ Trends Exp Clin Psychiatry 12: 243-252.

Burgess PW (1997). Theory and methodology in executive function research. In Rabbitt P (eds). Methodology of Frontal and Executive Function. Psychology Press: Hove. pp 81-116.

Callicott JH, Mattay VS, Bertolino A, Finn K, Coppola R, Frank JA et al (1999). Physiological characteristics of capacity constraints in working memory as revealed by functional MRI. Cerebr Cortex 9: 20-26.

Callicott JH, Ramsey NF, Tallent K, Bertolino A, Knable MB, Coppola $\mathrm{R}$ et al (1998). Functional Magnetic Resonance Imaging brain mapping in psychiatry: methodological issues illustrated in a study of working memory in schizophrenia. Neuropsychopharmacology 18: 186-196.

Carlsson A, Hansson LO, Waters N, Carlsson ML (1999). A glutamatergic deficiency model of schizophrenia. Br J Psychiatry 174(Suppl 37): 2-6.

Carter CS, Perlstein W, Ganguli R, Brar J, Mintun M, Cohen JD (1998). Functional hypofrontality and working memory dysfunction in schizophrenia. Am J Psychiatry 155: 1285-1287.

Cocosco CA, Kollokian V, Kwan RKS, Evans AC (1997). Brainweb: online interface to a 3D MRI simulated brain database. Neuroimage 5: 425.

Cohen JD, Perlstein WM, Braver TS, Nystrom LE, Noll DC, Jonides $\mathrm{J}$ et al (1997). Temporal dynamics of brain activation during a working memory task. Nature 386: 604-608.

Conklin HM, Curtis CE, Katsanis J, Iacono WG (2000). Verbal working memory impairment in schizophrenia patients and their first-degree relatives: evidence from the digit span task. Am J Psychiatry 157: 275-277.

Curtis VA, Bullmore ET, Brammer MJ, Wright IC, Williams SC, Sharma TS et al (1998). Attenuated frontal activation during a verbal fluency task in patients with schizophrenia. $A m J$ Psychiatry 155: 1056-1063.

D’Esposito M, Postle BR, Ballard D, Lease J (1999). Maintenance versus manipulation of information held in working memory: an event-related fMRI study. Brain Cognition 41: 66-86.

De Zubicaray GI, Williams SCR, Wilson SJ, Rose SE, Brammer MJ, Bullmore ET et al (1998). Prefrontal cortex involvement in selective letter generation: a functional magnetic resonance imaging study. Cortex 34: 389-401.

Domino EF, Zsigmond EK, Domino LE, Domino KE, Kothary SP, Domino SE (1982). Plasma levels of ketamine and two of its metabolites in surgical patients using a gas chromatographic mass fragmentographic assay. Anesthesia Analgesia 61: 87-92.

Fletcher PC, Henson RNA (2001). Frontal lobes and human memory: insights from functional neuroimaging. Brain 124: 849-881.

Friedman HR, Goldman-Rakic PS (1994). Coactivation of prefrontal cortex and inferior parietal cortex in working memory tasks revealed by 2DG functional mapping in the rhesus monkey. J Neurosci 14: 2775-2788.

Friston KJ, Holmes AP, Worsley KJ, Poline J-B, Frith CD, Frackowiak RSJ (1995). Statistical parametric maps in functional imaging: a general linear approach. Hum Brain Mapp 2: 189-210.

Genovese CR, Lazar NA, Nichols T (2002). Thresholding of statistical maps in functional neuroimaging using the false discovery rate. Neuroimage 15: $870-878$.
Gepts E (1998). Pharmacokinetic concepts for TCI anaesthesia. Anaesthesia 53(Suppl 1): 4-12.

Ghoneim MM, Hinrichs JV, Mewaldt SP, Petersen RC (1985). Ketamine: behavioral effects of subanesthetic doses. J Clin Psychopharmacol 5: 70-77.

Gold JM, Harvey PD (1993). Cognitive deficits in schizophrenia. Psychiatr Clin N Am 16: 295-312.

Goldman-Rakic PS (1996). The prefrontal landscape: implications of functional architecture for understanding human mentation and the central executive. Philosoph Trans R Soc London B 351: $1445-1453$.

Gooding DC, Tallent KA (2002). Spatial working memory performance in patients with schizoaffective psychosis versus schizophrenia: a tale of two disorders? Schizophrenia Res 53: 209-218.

Hirsch SR, Das I, Garey LJ, deBelleroche J (1997). A pivotal role for glutamate in the pathogenesis of schizophrenia, and its cognitive dysfunction. Pharmacol Biochem Behavr 56: 797-802.

Holcomb HH, Lahti AC, Medoff DR, Weiler M, Tamminga CA (2001). Sequential regional cerebral blood flow brain scans using PET with $\mathrm{H}_{2}^{15} \mathrm{O}$ demonstrate ketamine actions in CNS dynamically. Neuropsychopharmacology 25: 165-172.

Honey RAE, Turner DC, Honey GD, Sharar SR, Kumaran D, Pomarol-Clotet E et al (2003). Low dose ketamine produces a deficit in manipulation but not maintenance of the contents of working memory. Neuropsychopharmacology 28: 2037-2044.

Javitt DC, Zukin SR (1991). Recent advances in the phencyclidine model of schizophrenia. Am J Psychiatry 148: 1301-1308.

Jentsch JD, Roth RH (1999). The neuropsychopharmacology of phencyclidine: from NMDA receptor hypofunction to the dopamine hypothesis of schizophrenia. Neuropsychopharmacology 20: 201-225.

Jonides S, Schumacher EH, Smith EE, Lauber EJ, Awh E, Minosiima J et al (1997). Verbal working memory load affects regional brain activation as measured by PET. J Cogni Neurosci 9: $462-475$.

Kegeles LS, Abir-Dargham A, Zea-Ponce Y, Rodenhiser-Hill J, Mann JJ, Van Heertum RL et al (2000). Modulation of amphetamine-induced striatal dopamine release by ketamine in humans: implications for schizophrenia. Biol Psychiatry 48: 627-640.

Kharasch ED, Labroo R (1992). Metabolism of ketamine stereoisomers by human liver microsomes. Anesthesiology 77: 12011207.

Krystal JH, Abi-Dargham A, Laruelle M, Moghaddam B (1999). Pharmacologic models of psychoses. In Charney DS, Nestler EJ, Bunney BS (eds). Neurobiology of Mental Illness, pp 214-224, Oxford University Press: New York, NY.

Krystal JH, Karper LP, Seibyl JP, Freeman GK, Delaney R, Bremner JD et al (1994). Subanesthetic effects of the noncompetitive NMDA antagonist, ketamine, in humans. Psychotomimetic, perceptual, cognitive, and neuroendocrine responses. Arch Gen Psychiatry 51: 199-214.

Lahti AC, Koffel B, LaPorte D, Tamminga CA (1995). Subanesthetic doses of ketamine stimulate psychosis in schizophrenia. Neuropsychopharmacology 13: 9-19.

Mahurin RK, Velligan DI, Miller AL (1998). Executive-frontal lobe cognitive dysfunction in schizophrenia: a symptom subtype analysis. Psychiatry Res 79: 139-149.

Manoach DS, Gollub RL, Benson ES, Searl MM, Goff DC, Halpern E et al (2000). Schizophrenic subjects show aberrant fMRI activation of dorsolateral prefrontal cortex and basal ganglia during working memory performance. Biol Psychiatry 48: 99-109.

Manoach DS, Press DZ, Thangaraj V, Searl MM, Goff DC, Halpern $\mathrm{E}$ et al (1999). Schizophrenic subjects activate dorsolateral prefrontal cortex during a working memory task, as measured by fMRI. Biol Psychiatry 45: 1128-1137. 
Menon V, Anagnoson RT, Mathalon DH, Glover GH, Pfefferbaum A (2001). Functional neuroanatomy of auditory working memory in schizophrenia: relation to positive and negative symptoms. NeuroImage 13: 433-446.

Moghaddam B, Adams B, Verma A, Daly D (1997). Activation of glutamatergic neurotransmission by ketamine: a novel step in the pathway from NMDA receptor blockade to dopaminergic and cognitive disruptions associated with the prefrontal cortex. J Neurosci 17: 2921-2927.

Morice R, Delahunty A (1996). Frontal/executive impairments in schizophrenia. Schizophrenia Bull 22: 125-137.

Nelson HE (1991). National Adult Reading Test. NFER-Nelson Publishing: Berkshire, UK.

Olney JW, Farber NB (1995). Glutamate receptor dysfunction and schizophrenia. Arch of Gen Psychiatry 52: 998-1007.

Oye I, Paulsen O, Maurset A (1992). Effects of ketamine on sensory perception: evidence for a role of $N$-methyl-D-aspartate receptors. J Pharmacol Exp Ther 260: 1209-1213.

Pantelis C, Barnes TRE, Nelson HE, Tanner S, Weatherley L, Owen $\mathrm{AM}$ et al (1997). Frontal-striatal cognitive deficits in patients with chronic schizophrenia. Brain 120: 1823-1843.

Park S, Holzman PS (1992). Schizophrenics show spatial working memory deficits. Arch Gen Psychiatry 49: 975-982.

Postle BR, Berger JS, D’Esposito M (1999). Functional neuroanatomical double dissociation of mnemonic and executive control processes contributing to working memory performance. Proc Natl Acad Science USA 96: 12959-12964.
Smith EE, Jonides J, Koeppe RA (1996). Dissociating verbal and spatial working memory using PET. Cerebr Cortex 6: $11-20$.

Smith GS, Schloesser R, Brodie JD, Dewey SL, Logan J, Vitkun SA et al (1998). Glutamate modulation of dopamine measured in vivo with positron emission tomography (PET) and ${ }^{11} \mathrm{C}$-raclopride in normal human subjects. Neuropsychopharmacology 18: 18-25.

Tamminga C (1999). Glutamatergic aspects of schizophrenia. $\mathrm{Br} J$ Psychiatry 174(Suppl 37): 12-15.

Tamminga CA (1998). Schizophrenia and glutamatergic transmission. Crit Rev Neurobiol 12: 21-36.

Tek C, Gold J, Blaxton T, Wilk C, McMahon RP, Buchanan RW (2002). Visual perceptual and working memory impairments in schizophrenia. Arch Gen Psychiatry 59: 146-153.

Tsai G, Coyle JT (2002). Glutamatergic mechanisms in schizophrenia. Ann Rev Pharmacol Toxicol 42: 165-179.

Vollenweider FX, Leenders KL, Oye I, Hell D, Angst J (1997a). Differential psychopathology and patterns of cerebral glucose utilisation produced by (S)- and (R)-ketamine in healthy volunteers using positron emission tomography (PET). Eur Neuropsychopharmacol 7: 25-38.

Vollenweider FX, Leenders KL, Scharfetter C, Antonini A, Maguire $\mathrm{P}$, Missimer J et al (1997b). Metabolic hyperfrontality and psychopathology in the ketamine model of psychosis using postiron emission tomography (PET) and $\left[{ }^{18} \mathrm{~F}\right]$ fluorodeoxyglucose (FDG). Eur Neuropsychopharmacol 7: 9-24. 